\title{
Interview: Omnia vincit amor. Pectus est quod facit disertos. Ein Gespräch mit Prof. Dr. Drs. h.c. Ulrich Engel anlässlich seines 80 . Geburtstages
}

Joanna Golonka, Mariola Wierzbicka: In Ihrem Beitrag zum Konferenzband anlässlich des 60. Geburtstags von Prof. Zdzisław Wawrzyniak haben Sie nach eigenen Worten - ,eine Art Quintessenz Ihres Linguistendaseins“ formuliert. Nun steht Ihnen Ihr eigenes Jubiläum bevor: Im November dieses Jahres feierten Sie Ihren 80. Geburtstag. So ein Anlass bewegt Sie sicher dazu, öfters auf Ihr Leben zurückzuschauen.

Ulrich Engel: Mein Leben ist keineswegs besonders interessant für andere, und ich halte es auch nicht für irgendwie vorbildlich. Aber als exemplarisches Leben erscheint es mir in der Rückschau schon, und daraus können später Geborene vielleicht etwas lernen.

J.G.: Dürften wir Sie bitten, uns einiges aus Ihrem Leben zu erzählen? Sie waren ein Kind, als in Deutschland die folgenschwere Hitler-Ära begann.

U.E.: Ich war, als Hitler an die Macht kam, vier Jahre und zwei Monate alt und damit den Einflüssen jener Zeit umso mehr ausgeliefert, als das Elternhaus mir keinerlei Unabhängigkeit sicherte. Zwar waren die Eltern gutbürgerlicher Herkunft, und das hieß in jener Zeit meist: deutschnational geprägt. Aber meine sehr junge Mutter verfiel schnell der Sozialpropaganda der neuen Herrscher (dieses Register haben die Nationalsozialisten meisterlich genutzt), und der Vater gehörte zu den ,Mai-Gefallenen“, die im Mai 1933 in die NSDAP eintraten, dem spätesten Zeitpunkt, zu dem der Parteieintritt noch kein Odium hatte. Was wäre ihm als Staatsbeamtem auch viel anderes übrig geblieben? Er wollte Karriere machen, er wollte keinen Krieg, keine Aggressionen, keine Verfolgungen, und die dominante Meinung des Jahres 1933 war, allen Übergriffen zum Trotz: Es wird schon nicht so schlimm werden, die werden auch nicht ewig an der Macht bleiben. In dieser Atmosphäre wurde ich zum Hitlerjungen, sehnte mich danach, die Uniform des ,Jungvolks‘ tragen zu dürfen. Wir waren ziemlich glücklich damals, wir sangen, machten gruppenweise Fahrten, veranstalteten Zeltlager, saßen abends um das verglimmende Feuer. Die Welt wollten wir nicht erobern (auch wenn wir davon gelegentlich sangen), besser machen vielleicht schon, und wir waren ganz sicher, dass uns das gelingen würde. 
M.W.: Wie haben Sie die Atmosphäre jener Zeit persönlich mitempfunden?

U.E.: Ich bin ein Kind der süddeutschen Provinz, das lässt manches harmloser erscheinen. Bei uns durfte man schon mal über einen Funktionär der Partei schimpfen, ohne denunziert oder gar verhaftet zu werden. Die Großstädte waren straffer durchorganisiert, da musste man seine Zunge hüten; aber in den Stuttgarter Arbeitervierteln wurden auch Hitlerjungen gelegentlich blutig geschlagen. In meiner kleinen ostschwäbischen Landstadt, einer einstigen Reichsstadt, der jedes urbane Flair abging, sorgte man sich ums tägliche Brot und das sonntägliche Fleisch wie vordem, meine Klassenkameraden gingen zur Nachhilfe zu einem entlassenen Lehrer, einem früheren Kommunisten, während dessen Frau der neu gebildeten parteigeförderten , Kindergruppe ' vorstand. Die Judenhetze bekamen wir wohl mit, aber da das deutsche Bürgertum damals weithin antisemitisch geprägt war, löste dies keine Proteste aus, auch keine heimlichen, und das fühlbare Verschwinden der jüdischen Mitbürger bis hin zur ,Kristallnacht' des Jahres 1938 registrierte man ohne weiteres Nachdenken. Niemand dachte an Massenmord, es war ja auch bequemer so.

J.G.: Brachte der Kriegsausbruch eine Änderung Ihrer Lebensführung mit sich? Welche Folgen hatte der von Hitler angezettelte totale Krieg für Sie?

U.E.: Zunächst ging es in der geschilderten Art weiter. Es muss euch schwerfallen, das zu glauben: Wir waren die Hitler treu ergebene Jugend, wir bewunderten ihn und nahezu alles, was unter seiner Ägide geschah. Wir verfolgten die deutschen Eroberungen, viele Familien hatten sich Europakarten aufgehängt, und die Jungen steckten Fähnchen ein bei den Städten und Landstrichen, zu denen die deutschen Truppen vorgedrungen waren. Was mit den Leuten dort geschah, erfuhren wir nicht, jedenfalls nicht zuverlässig, und es interessierte uns fanatisierte Kinder auch nicht weiter. So bekamen wir auch die Bedeutung von Stalingrad, dem eigentlichen Fanal der Kriegswende, nicht richtig mit. Goebbels, den keiner mochte, über den man sogar Witze machte, begeisterte uns dennoch mit seiner Durchhalterede vom „totalen Krieg“ im Februar 1943 im Berliner Sportpalast. Meine Lebensführung entpolitisierte sich erst dann radikal, als wir zum Militär eingezogen wurden. Wir waren fünfzehn Jahre alt und sollten Industrieanlagen um die Stadt Friedrichshafen am Bodensee gegen feindliche Bombenangriffe verteidigen. Ich selbst war körperlich schwächlich und sollte zurückgestellt werden; erst nachdem mein Vater auf meine dringende Bitte und mit gemischten Gefühlen den Stabsarzt um eine nochmalige Musterung gebeten hatte, durfte ich mich den Altersgenossen anschließen. Jetzt endlich waren wir Männer, rauchten, tranken gele- 
gentlich auch Alkohol, terrorisierten unsere Lehrer, die in der Flak-Batterie noch Unterricht geben mussten. Ein ,Politischer Leiter', wie die Funktionäre damals hießen, hätte bei uns keinen Ansatzpunkt gefunden. Es wurde sogar massive antinazistische Kritik laut nachts in der Wohnbaracke, und niemand hätte die Feinde des ,Führers' verraten. Ein Bombenangriff auf unsere Stellung im Sommer 1944, bei dem 22 meiner Altersgenossen umkamen, brachte Entsetzen unter uns, aber immer noch keine Zweifel am ,Endsieg', und so kam der Zusammenbruch im Allgäu und der Übergang in französische Gefangenschaft, der mich die Taschenuhr meines Urgroßvaters kostete, wie ein unbegreiflicher Schicksalsschlag über uns. Ein paar Tage verbrachten wir in Immenstadt in einem Kinosaal. Ein ehemaliger Kriegsgefangener verkündete uns vor der Leinwand: „Krieg aus, Hitler tot. In sechs Wochen alles heim.“ Und der Saal brüllte vor Freude.

M.W.: Bedeuteten diese Worte für Sie wirklich das Ende der Kriegserfahrungen?

U.E.: Irgendwie schon. Zunächst fühlten wir uns, und das war völlig ungewohnt, machtlos und müde. In endlosen Märschen liefen wir, nachts von Panzerwagen mit Scheinwerfern bewacht, dem Bodensee, dann dem Hegau zu. Von dort wurden wir auf offenen Lastwagen in rasender Fahrt durch den Schwarzwald ins Lager Kehl gebracht. An den Straßen standen Frauen, die weinten und uns beklagten und Brotlaibe auf den Wagen warfen. Ich stand ganz hinten und bekam das meiste ab, auch einmal einen Brotlaib ins Gesicht, das brannte wie Feuer. „Alles weitergeben, wir verteilen nachher“, riefen die weiter vorn Stehenden. Als wir in Kehl ankamen, hatten alle ihr Brot und manche noch Schinken dazu, ich allein hatte nichts und war auf gutherzige Spender angewiesen. Wir kamen tief nach Frankreich und hatten im Wald, im Steinbruch, auf dem Friedhof zu arbeiten. Ich erhielt eine leichtere Arbeit und kam, da ich unterernährt aussah (ausgehungert waren wir alle), schon nach neun Monaten frei. Inzwischen war ich siebzehn Jahre alt. Meine Altersgenossen Albert, Erich, Eugen, Gerhard blieben drei Jahre oder länger in Frankreich. Außer mir lebt heute nur noch Erich.

J.G.: Nun waren Sie zurück in Ihrer Heimat. Wie ging dort Ihr Leben weiter?

U.E.: In der Heimat fand ich alles verändert. Meine Mutter lebte mit fünf Kindern in einer Notwohnung auf dem Dorf, der Vater, zuletzt Landrat, war in , automatic arrest' genommen worden. Ich musste vieles neu lernen. Den Hitlergruß mit erhobenem rechtem Arm hatte ich zwar nie gegenüber Freunden und persönlichen Bekannten verwendet, auf Behörden war er jedoch Pflicht 
gewesen. Und ich musste nun viele Behördengänge machen, um in der neuen Umgebung leben zu dürfen. Ging ich aufs Rathaus, so sagte ich mir unterwegs fortwährend: ,Lass den rechten Arm unten!' Und trotzdem wurde ich die Angst nicht los, irgend einmal „Heil Hitler“ zu sagen.

M.W.: Wie haben Sie die Stimmung empfunden, die unter den Landsleuten in Ihrer Umgebung gleich nach dem Krieg vorherrschte?

U.E.: Die Einstellung großer Teile der deutschen Bevölkerung war damals so, dass man sich wenig mit Schuldfragen abgab. Persönliche Schuld hatte man nicht (oder glaubte sie wenigstens nicht zu haben), um eine deutsche Gesamtschuld aber wollte man sich nicht kümmern. Man konzentrierte vielmehr alle verbliebene Energie darauf, das zerstörte Land wieder aufzubauen. „Wir wollen es denen nochmal zeigen“, war für viele die Grundhaltung.

M.W.: Und wie ging Ihr Leben dann weiter? Wie haben Sie die restliche Zeit Ihrer Jugend verbracht? Hatten Sie auch schon konkrete Berufspläne?

U.E.: Die restlichen Schuljahre brachten mir teils Vergnügen, teils Frust. Ich wollte Lehrer werden, um besser zu machen, was meine Lehrer an uns verpatzt hatten. Aber mit dem Studium in Tübingen, zwischendurch zwei Semester in Göttingen, konnte ich nur teilweise etwas anfangen. Die Literaturwissenschaft, wie sie damals betrieben wurde, ödete mich an, nur die Sprachwissenschaft machte Freude. Und die damalige Ordinarienuniversität war ein gänzlich unverständliches Monster. Immerhin schaffte ich den Abschluss, die Promotion „sehr gut“, das Staatsexamen eben noch „,befriedigend“, und stürzte mich voller Elan in die Schularbeit. Ich glaube, dass ich gut war als Lehrer, denn ich zähmte die rebellischsten Klassen, erzielte überdurchschnittliche Leistungserfolge, und dennoch war ich bei den Schülern beliebt. Bei den Kollegen weniger, und sie, einige von ihnen jedenfalls, sind auch ein Grund dafür, dass ich nach zehn Jahren in die Wissenschaft wechselte.

\section{J.G.: Wie sah dieser Wechsel aus?}

U.E.: Im Frühjahr 1964 wurde, als mein Doktorvater Hugo Moser den Dudenpreis erhielt, gleichzeitig die Gründung eines Instituts für deutsche Sprache bekannt gegeben (eine jahrelang verfolgte Idee Mosers). Ich erklärte ihm damals, dass mich die Mitarbeit in einem solchen Institut reize. Er zeigte sich davon begeistert. So wechselte ich im April 1965 nach Mannheim, zuerst als Stellvertreter Paul Grebes, ab 1970 als erster Direktor. Dieses Institut wäre der ideale Ort für wissenschaftliche Arbeit gewesen, wenn mich das Direktorenamt nicht diesem Ziel zunehmend entfremdet hätte. Deshalb wollte ich, 
durchaus mit Einkommensminderung, in die Gilde der Mitarbeiter zurücktreten. Das war den Zuständigen bekannt. Die Umstände, unter denen dies im Juni 1976 geschah, waren nicht die von mir gewünschten. Es hätte sich damals leicht nachweisen lassen, dass nicht in erster Linie ein Versagen des Direktors (sowie der Verwaltungsspitze), sondern vor allem nicht eingelöste Zusagen der Geldgeber zur finanziellen Schieflage des Instituts geführt hatten. Mein Wunsch, diese Dinge offenzulegen, war gering, ich hatte ja keinen Besitzstand zu verteidigen, und ich hatte schließlich, wenngleich auf weniger angenehme Art, erreicht, was ich erreichen wollte.

M.W.: Also konnten Sie sich nun endlich ausschließlich der Wissenschaft und der Forschung widmen. Konnten Sie im IDS selbst Ihr Forschungsgebiet wählen? In welche Richtung sind Sie gegangen?

U.E.: Die Art, wie ich seither Wissenschaft betrieb, beruhte nur teilweise auf meiner eigenen Entscheidung. Tesnière zwar und die Dependenzgrammatik hatte ich mir, von Hennig Brinkmann angestoßen, gewissermaßen selbst ausgesucht. Aber eine Zeit lang war es vor allem die Neugier am Fremden, die mich fesselte. Was mich letztlich zum Dependenzgrammatiker werden ließ, war die wachsende Erkenntnis, dass dieses Grammatikmodell, richtig eingesetzt und angemessen fortentwickelt, allen anderen Modellen ebenbürtig und deshalb als vollgültige Alternative zu pflegen ist. Es muss in der Wissenschaft Alternativen geben. Dass diese Mannheimer Dependenzgrammatik vor allem in kontrastiven Darstellungen Anwendung fand, ist Zufall, ich habe das oft betont. Die Kulturabteilung des Auswärtigen Amtes wollte das Deutsche als Fremdsprache in der Welt fördern und suchte, da wissenschaftliche Grundlagen Ende der 60er Jahre fast völlig fehlten, zuverlässige linguistische Beschreibungen, die als Grundlage für Fremdsprachenlehrwerke dienen könnten. Nach dem Verständnis jener Zeit waren kontrastive Grammatiken geeignet, zur Optimierung des Fremdsprachenunterrichts beizutragen. Diese Annahme, auch wenn sie nach heute vorliegenden Erkenntnissen relativiert werden muss, hat es immerhin ermöglicht, moderne Fremdsprachenlehrwerke auf eine taugliche Referenzliteratur zu stützen. Es wäre ein Irrtum zu glauben, dass effektive Fremdsprachenlehrwerke ohne tieferen Einblick in die Beziehungen zwischen Ausgangs- und Zielsprache überhaupt möglich wären. Das IDS hatte also im Auftrag des Auswärtigen Amtes eine Reihe kontrastiver Grammatiken zu erarbeiten. Ich selbst musste - als Direktor, dann als vom Kuratorium schnell ernannter Leiter der Abteilung „Kontrastive Grammatik“ - diese Forschungsobjekte anerkennen, auch wenn mir damals Theorie- und Modell- 
vergleiche viel mehr am Herzen lagen. Gott sei Dank, sage ich heute, haben die Umstände damals gegen meine temporären Neigungen entschieden.

J.G.: Und wie hat es mit den Plänen über die deutsch-polnische kontrastive Grammatik angefangen, die Ihnen - wie ich im IDS und anderswo sowie auch von Ihnen selbst gehört habe - besonders am Herzen liegt?

U.E.: Dass meine kontrastiven Pläne auf Polen und das Polnische fielen, ist kein Zufall. Es hängt mit meiner politischen Entwicklung zusammen. Seit der ersten Bundestagswahl (1949) bin ich Sozialdemokrat. Das heißt, dass ich auf der Seite der Werktätigen, der Arbeitnehmer stehe und den Vertretern des Kapitals äußerst kritisch gegenüberstehe. Das heißt auch, dass ich mit einer kompromisslosen Westorientierung Deutschlands nie einverstanden war. Ich habe deshalb ständig Kontakte mit den Menschen im Sowjetblock gesucht, so 1958, als ich mit einer privaten Reise nach Zittau in Sachsen die Verkrustung der Beziehungen durchbrechen wollte; so 1967 in Rumänien, als ich abseits des Linguistenkongresses private Kontakte suchte. 1968 erlebte ich den Besuch vieler Polen, und damals entstand die Idee der kontrastiven deutsch-polnischen Grammatik. Widerstände kamen zum Teil aus dem Kuratorium des IDS, vor allem aber aus dem Auswärtigen Amt. Ein Jahr vor den Ostverträgen und Willi Brandts Kniefall erklärte man mir kategorisch, dass man eine deutsch-polnische Grammatik nicht wolle. Als neuer Geldgeber trat 1976 die Stiftung Volkswagenwerk auf den Plan. Sie hätte eine solche Grammatik gern gesehen, aber einwöchige Gespräche in Warschau scheiterten an politischen Vorbehalten der polnischen Seite. Dann war äußerlich Stille, es erschienen die deutschserbokroatische Grammatik (1986) und die deutsch-rumänische Grammatik (1993). Aus beiden war zu lernen, Positives und Negatives. Aber mein polnischer Traum lebte weiter. 1988 reiste ich durch Polen, aus eigener Initiative, aber mit Unterstützung des IDS, und suchte mir Mitarbeiter. Ich fand sie schnell und problemlos an den polnischen Universitäten Wrocław, Poznań, Katowice-Sosnowiec, Kraków, Rzeszów und Łódź. Mit ihnen zusammen schrieben wir die deutsch-polnische kontrastive Grammatik ( $d p g$ ), die endlich 1998 erschien. Es war ein langer Weg bis dahin. Heute sitzen wir, mit teilweise neuen Mitarbeitern, an der zweiten Auflage und an einem zusätzlichen kommunikativen Band. Mein polnischer Traum ist noch lange nicht ausgeträumt, man kann ihn mir dann ins Grab mitgeben. 
M.W.: Sie können auf ein reiches wissenschaftliches Leben zurückblicken. Sie haben viel in die Wege geleitet, viel erreicht und zahlreiche Wissenschaftler zu ihrem beruflichen Erfolg begleitet. Fühlen Sie sich als Wissenschaftler und Forscher, erfüllt'?

U.E.: Ich bin keineswegs zufrieden mit mir und meiner Arbeit. Ich möchte noch vieles tun, aber langsam lassen die Kräfte nach. Und mir ist auch klar geworden, dass die Sprachwissenschaft, die mir immer am Herzen lag, die mich seit meiner Dissertation nicht mehr losgelassen hat, nicht alles sein darf. Es gibt Dinge, die du, wenn du einer geworden bist, auf den sie hören, ebenfalls in die Hand nehmen musst. Ich möchte sie hier nicht alle aufzählen, soviel nur: Sie haben vorrangig mit Umweltschutz, mit Klimaschutz, mit Tierschutz zu tun. Dafür arbeite ich seit über zehn Jahren als Sprecher der Lokalen Agenda 21 Heppenheim, der einzigen von zahlreichen gleichartigen Bewegungen, die im Kreis Bergstraße überlebt hat.

J.G.: Was würden Sie jüngeren Kolleginnen und Kollegen auf den Weg geben?

U.E.: Ich verfüge nicht über Lebensweisheiten, die ich weitergeben könnte. Ich habe auch nicht immer vernünftig gelebt. Wenn es euch aber um ein paar Verhaltensempfehlungen geht, so würde ich sagen: Mach nach Möglichkeit nur, was du mit Freude machst. Mach das, was du besser kannst als andere. Und wenn es dazuhin noch anderen nützt, dann wird es gut sein. 